\title{
Tratamento da hérnia perineal com uso de tela de polipropileno revestida de metilcelulose: relato de caso
}

\author{
Treatment of perineal hernia using interposition of a metylcelullosis encapsulated \\ mesh: case report
}

\author{
DULCYANE FERREIRA DE OLIVEIRA'; CARLOS HENRIQUE MARQUES DOS SANTOS²; LÊNIN DE LIMA \\ RODRIGUES ${ }^{3}$; MÁRCIO EDUARDO DE SOUZA PEREIRA ${ }^{4}$
}

\begin{abstract}
${ }^{1}$ Acadêmica do $6^{\circ}$ ano de Medicina da Universidade Federal de Mato Grosso do Sul (UFMS); Membro da Liga Acadêmica de Cirurgia (LAC) - Campo Grande (MS), Brasil. ${ }^{2}$ Doutor em Medicina; Professor Titular da Sociedade Brasileira de Coloproctologia (TSBCP); Professor Titular do Colégio Brasileiro de Cirurgiões (TCBC); Cirurgião Geral do Hospital Regional de Mato Grosso do Sul - Campo Grande (MS), Brasil. ${ }^{3}$ Médico; Cirurgião Geral do Hospital Geral do Exército de Campo Grande - Campo Grande (MS), Brasil. ${ }^{4}$ Médico; Especialista em Cirurgia Digestiva e Videocirurgia do Hospital Regional de Mato Grosso do Sul - Campo Grande (MS), Brasil.
\end{abstract}

OLIVEIRA DF; SANTOS CHM; RODRIGUES LL; PEREIRA MES. Tratamento da hérnia perineal com uso de tela de polipropileno revestida de metilcelulose: relato de caso. Rev bras Coloproct, 2011;30(4): 455-458.

RESUMO: A incidência de hérnia perineal (HP) é menor que 1\% dos pacientes submetidos à amputação abdominoperineal do reto (AAPR). O objetivo é relatar um caso de HP pós AAPR por adenocarcinoma de reto distal. É descrito o caso de uma paciente de 56 anos que, após cirurgia de AAPR, apresentou precocemente HP. O tratamento consistiu em redução do conteúdo herniário e colocação de uma tela de polipropileno revestida de metilcelulose. Por ser afecção rara e apresentar etiologia variada, o tratamento não apresenta consenso, havendo inúmeras técnicas descritas. Conclui-se que o uso da prótese sintética para correção de HP é satisfatório e permite a obtenção de bons resultados.

Descritores: Hérnia; Períneo; Telas cirúrgicas; Complicações pós-operatórias.

\section{INTRODUÇÃO}

As hérnias perineais (HP) podem ser definidas como a protrusão de uma massa entre os músculos e fáscias que formam o piso do períneo. Podem ser classificadas em primárias ou secundárias, estando relacionadas a vários fatores etiopatogênicos, tais quais: ressecção do cóccix, histerectomia e excisão dos músculos elevadores do ânus - procedimentos costumeiramente realizados na técnica operatória de Miles e na condução de cânceres colorretais ${ }^{1}$.

A amputação abdominoperineal do reto (AAPR) é uma cirurgia de grande porte e com índices de com- plicações pós-operatórias elevados, estando a hérnia perineal presente em $0,6 \%$ dos casos ${ }^{2}$.

A hérnia e seu reparo cirúrgico são temas extensamente estudados e de profundo interesse por parte dos cirurgiões, e por isso as correções estão distantes de um consenso ${ }^{3}$. Assim, por ser uma patologia de baixa incidência e ampla etiologia, a HP apresenta inúmeras técnicas corretivas descritas.

\section{RELATO DO CASO}

É descrito o caso de uma paciente de 56 anos de idade que, após ser submetida à AAPR, evoluiu no ter-

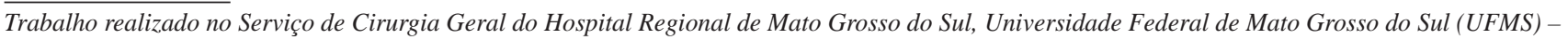
Campo Grande (MS), Brasil..

Recebido em: $12 / 8 / 2010$

Aceito para publicação em: 8/9/2010 
ceiro mês pós-operatório com abaulamento em períneo posterior associado a desconforto local (Figura 1).

Diagnosticada ao exame clínico hérnia perineal, a paciente foi submetida ao tratamento cirúrgico, sendo a via de acesso escolhida a perineal, através de incisão longitudinal na região glútea.

Seguiram-se os mesmos princípios de correção das demais hérnias: abertura por planos (Figura 2), mobilização do saco herniário (Figura 3), redução de seu conteúdo, excisão do saco e fechamento (Figura 4); porém, inovou-se ao optar pela interposição de uma tela de polipropileno revestida de metilcelulose entre o peritônio e as vísceras, fixando-a ao periósteo, considerando que não havia musculatura para aproximação (Figura 5).

A paciente evoluiu satisfatoriamente, sem sinais de recidiva dez meses após o procedimento.

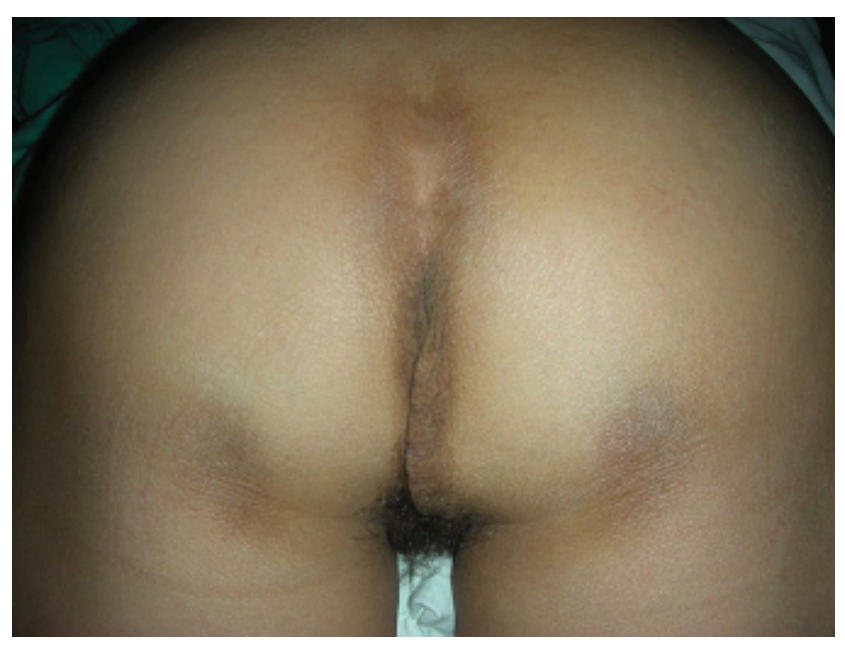

Figura 1 - Paciente em decúbito dorsal apresentando abaulamento em sulco interglúteo, borda lateral esquerda.

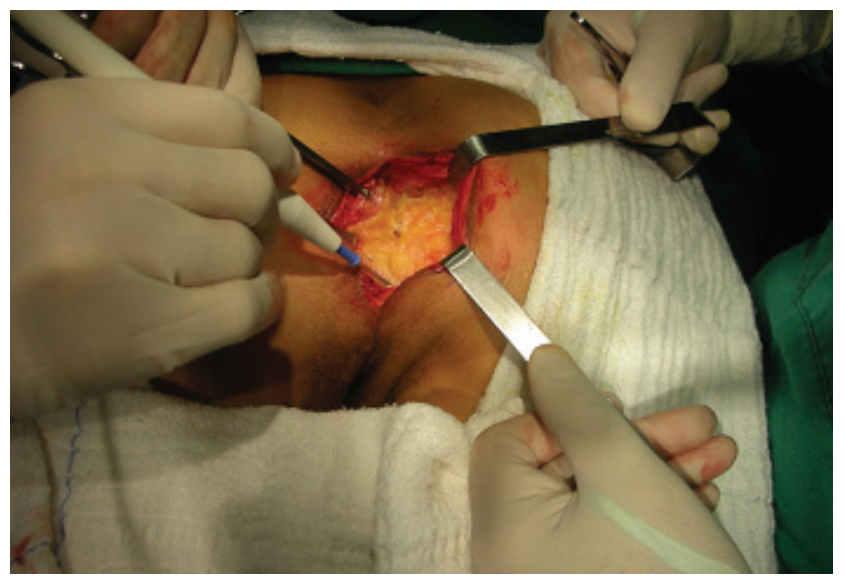

Figura 2 - Abertura da pele e tecido celular subcutâneo.

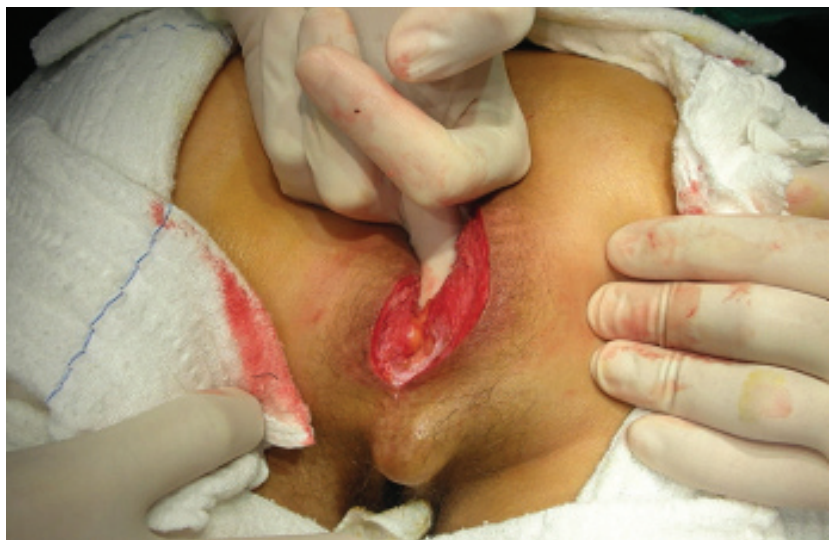

Figura 3 - Mobilização do saco herniário.

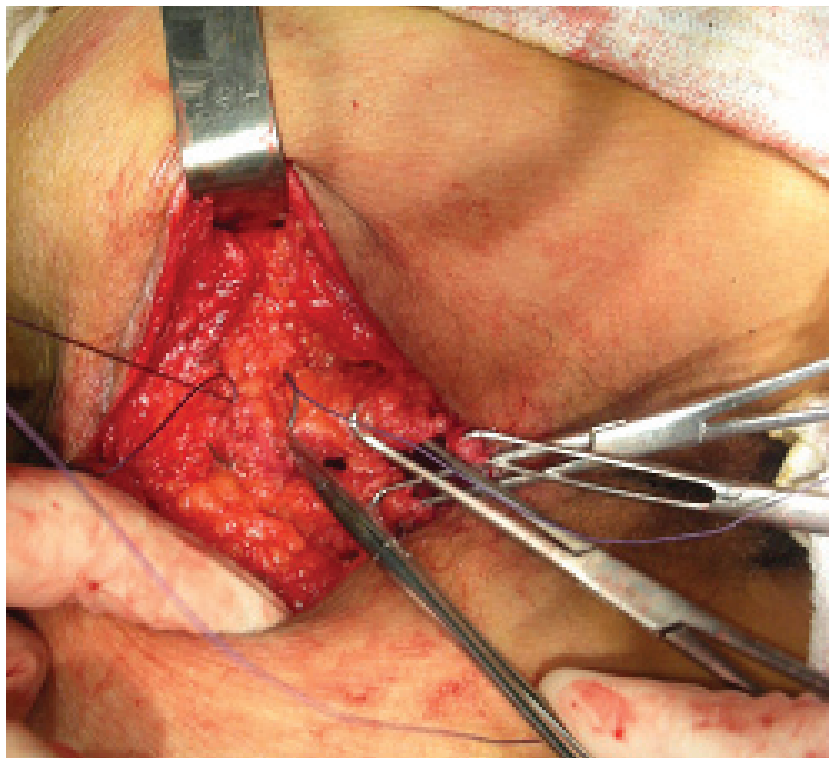

Figura 4 - Fechamento do saco herniário.

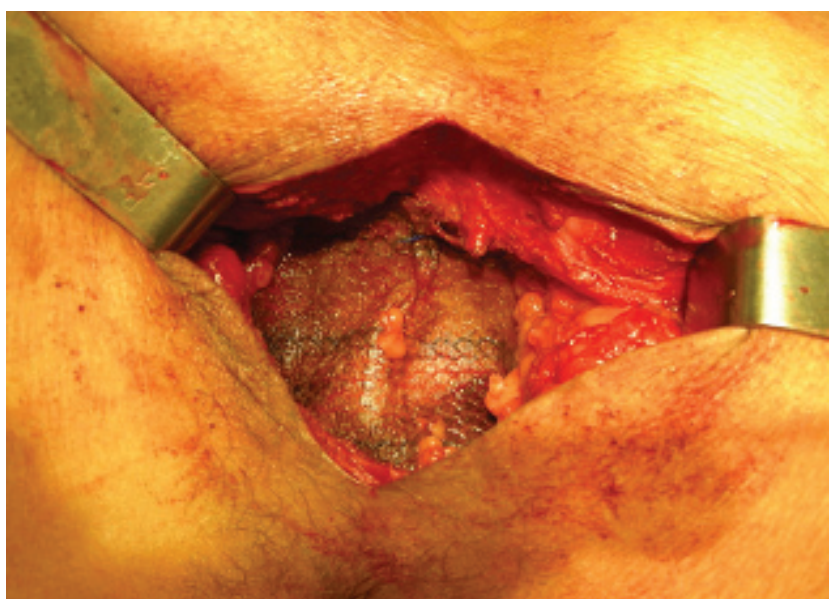

Figura 5 - Fixação da tela de polipropileno revestida de metilcelulose. 


\section{DISCUSSÃO}

As HP podem ser definidas como um defeito no assoalho pélvico que permite a passagem de conteúdo abdominal para a região pelve-perineal ${ }^{3,4}$. Acometem pacientes entre a quinta e a sétima décadas de vida, sobretudo mulheres, em uma proporção de 5:1 $1^{3,5}$. A incidência de HP sintomáticas, secundárias a uma cirurgia abdominoperineal, tem sido apresentada em $0.62^{6}$ a $1 \%{ }^{7,8}$ dos casos.

O quadro clínico é geralmente caracterizado por desconforto perineal ao sentar, sensação de peso perineal, laceração da pele, sintomas urinários e obstrução intestinal ${ }^{6}$, sendo o diagnóstico costumeiramente estabelecido já na história clínica e exame físico. Eventualmente são necessários exames de imagem como radiografia contrastada, tomografia ou ressonância magnética, estabelecendo-se diferenciação com lipomas, fibromas, retocele, cistocele e prolapso do reto ${ }^{3-5}$.

Pacientes idosos e pouco sintomáticos podem apresentar alívio apenas com uso de bandagens locais, não sendo necessária a cirurgia corretora.

O procedimento pode ser realizado por via perineal, abdominal ou videolaparoscópica; contudo, a reparação desse tipo de defeito ainda se apresenta como um desafio cirúrgico no qual vários métodos foram descritos, mas a abordagem ideal ainda tem de ser estabelecida ${ }^{6,7}$.

Em um estudo no Hospital Geral de Massachusetts com 21 pacientes tratados, entre 1963 e 1995, de HP pós-operatória, concluiu-se que a melhor via de abordagem é a perineal. é uma via atrativa porque não requer abertura do abdome nem anestesia geral, e pode resolver o problema de uma forma menos agres- siva. Entretanto, pode haver alguma dificuldade na liberação de aderências intestinais ${ }^{6}$.

Retalhos de glúteo máximo e reto abdominal foram descritos por alguns autores, mas tais técnicas aumentam a morbidade do procedimento e não apresentam resultados superiores aos de outras.

A retroflexão e fixação do útero podem ser uma boa alternativa via perineal, assim como o fechamento primário dos músculos elevadores. Entretanto, é comum encontrarmos pacientes já histerectomizadas e sem músculos elevadores, considerando a radicalidade dada à cirurgia prévia, de modo que a colocação de próteses sintéticas passa a ser a alternativa com maior chance de bons resultados.

As telas geralmente utilizadas para correção de hérnias são de polipropileno (PP) e de politetrafluoroetileno expandido (PTFE) ${ }^{9}$, contudo, o uso da tela de polipropileno revestida de metilcelulose mostra-se adequada porque contém em seu lado parietal PROLENE* Soft encapsulada com polidioxanona absorvível e, em sua face visceral, celulose oxidada regenerada, formando um gel que separa fisicamente a tela dos tecidos adjacentes e superfícies dos órgãos, o que diminui consideravelmente a chance de formação de aderências. Além disso, dentro de sete a dez dias, um neoperitônio é formado e, logo no $14^{\circ}$ dia, a tela já está absorvida ${ }^{10}$ e o peritônio totalmente reconstituído, aumentando o sucesso da reparação cirúrgica nos casos selecionados para sua aplicação.

\section{CONCLUSÃO}

Concluímos que o uso da prótese de polipropileno revestida de metilcelulose para correção de HP é satisfatório, permitindo a obtenção de bons resultados.

\footnotetext{
ABSTRACT: The incidence of perineal hernia (PH) is less than $1 \%$ among patients submitted to abdominoperineal resection. Our objective is to describe a case of $\mathrm{PH}$ after this procedure due to distal rectal adenocarcinoma. We report a case of a 56-year-old woman who presented with early PH. The treatment consisted of reduction of the hernia content and interposition of a methylcellulosisencapsulated mesh. Due to rarity and multi-etiology of this entity, there is no consensus about its best treatment, as many techniques have been described. We concluded that using a synthetic prosthesis is a satisfactory option to correct perineal hernias with good outcomes.
}

Key words: Hernia; Perineum; Surgical mesh; Postoperative complications. 


\section{REFERÊNCIAS}

1. Sobral HAC, Wolf JS, Carvalho RB, Lopes JM, Formiga GJS. Hérnia perineal primária: relato de caso. Rev Bras Coloproctol 2009;29(1):102-5.

2. Cruz GMG, Ferreira RMRS, Neves PM. Estudo Retrospectivo de 47 Complicações em 380 Pacientes Operados de Câncer Retal. Rev Bras Coloproctol 2006;26(2):138-55.

3. Thomford NR, Sherman NJ. Primary perineal hernia. Dis Colon Rectum 1969;12(6):441-3.

4. Mandarano R, Giorgi G, Venturini N, Mancini E, Natale A, Tiburzi C. Ernia perineale. Minerva Chir 1999;54 (7-8):523-9.

5. Salum MR, Prado-Kobata MH, Saad SS, Matos D. Primary perineal posterior hernia. An abdominoperineal approach for mesh repair of the pelvic floor. Clinics 2005;60(1):71-4.

6. So JB, Palmer MT, Shellito PC. Postoperative perineal hernia. Dis Colon Rectum 1997;40(8):954-7.

7. Beck DE, Fazio VW, Jagelman DG. Postoperative perineal hernia. Dis Colon Rectum 1987;30(1):21-4.
8. Sarr MG, Stewart JR, Cameron JC. Combined abdominoperineal approach to repair of postoperative perineal hernia. Dis Colon Rectum 1982;25(6):597-9.

9. Aydos RD, Silva IS, Goldenberg S, Goldenberg A, Simões MJ, Takita LC, et al. Estudo comparativo do efeito das telas de politetrafluoroetileno expandido e de polipropileno, colocadas por laparoscopia, em hérnias ventrais produzidas em coelhos. Acta Cir Bras 1999;14(2):59-64.

10. Proceed Chirurgische Mesh. Ethicon, Inc. A Johnson \& Johnson company somerville, New Jersey 2003. 2d barcode placement 389659.r03. [Cited July 2010]. Avaliable from: http://www.ethicon.de/netze/downloads/ (PROCEED)389659.R03.pdf

Endereço para correspondência: DULCYANE FERREIRA DE OLIVEIRA

Rua Guaporé, 111B - Ipiranga CEP 79080-750 - Campo Grande (MS), Brasil.

E-mail: dumeduf2004@yahoo.com.br Tel.: (67) 8406-5636/3346-2808 / (65) 9606-3647 